\title{
S. V. Znamenskij \\ A picture of common subsequence length for two random strings over an alphabet of 4 symbols
}

\begin{abstract}
The maximal length of longest common subsequence (LCS) for a couple of random finite sequences over an alphabet of 4 characters was considered as a random function of the sequences lengths $m$ and $n$. Exact probability distributions tables are presented for all couples of length in a range $2<m+n<19$.

The graphs of expected value and standard deviation as a functions of length are shown in linear perspective which presents the behaviour of large lengths at the horizon. In order to illustrate behaviour on large lengths, the results of numeric simulation for $m+n=32,512,8192$ and 131072 are also shown on the same graphs. The presented graph of expected value dependency of $m$ and $n$ looks to have asymptotic right circular cone. The variance looks alike growing as $(n+m)^{\frac{3}{4}}$.
\end{abstract}

Key words and phrases: similarity of strings, sequence alignment, edit distance, LCS, Levenshtein metric.

2010 Mathematics Subject Classification: 68T37; 68P10, 68W32.

The need for a theoretical estimation of occasional wrong match probability in fussy search with longest common subsequence (LCS) related algorithm raises the difficult problem of estimation the LCS length of two random symbol strings.

For practically important for applications in bioinfiormatics case of alphabet $\Sigma_{4}=A, C, G, T$ [1] it was found in [2] that natural DNAsequences from a database and true random sequences show the same statistical behavior in terms of similarity scores. The aim of this paper is to get a brief overall picture of functional dependency from both length of strings instead in spite of know for binary alphabet results [3].

Let's random variable $L_{m, n}$ notes the length of the longest common subsequence of random strings over the alphabet $\Sigma$ with lengths $m$ and $n$. The behaviour of its expected value $E\left(L_{m, n}\right)$ and variance $V\left(L_{m, n}\right)$ as a function of $m$ and $n$ remains dark and unclear for many decades. After [4] investigations for this alphabet are mostly concerned on bounding of

(c) S. V. ZnAMENSKiJ, 2016

(C) Ailamazyan Program System Institute of RAS, 2016

(C) Program systems: Theory and Applications, 2016 
Chvátal-Sankoff constant $\gamma_{4}=\lim _{n \rightarrow \infty} \frac{E\left(L_{n, n}\right)}{n}$ and detection of convergence rate for the limit value [5].

\section{Exact probability mass function}

We start from calculation of the exact probability mass function

$$
f_{L_{m, n}}(k)=P\left(L_{m, n}=k\right),
$$

where $P$ is probability for random variable $L_{m, n}$ to get a value $k=$ $0,1, \ldots, \min (m, n)$.

Theorem 1. Let $m<n$ are natural numbers. In the following simple cases the probability mass function $f_{L_{m, n}}(k)$ can be calculated by formulae:

$$
\begin{aligned}
& p_{1, n}(0)=\left(\frac{3}{4}\right)^{n} ; \\
& p_{1, n}(1)=1-\left(\frac{3}{4}\right)^{n} ; \\
& p_{2, n}(0)=\frac{1}{4}\left(\frac{3}{4}\right)^{n}+\frac{3}{2^{n+2}} \text { for } n>1 ; \\
& p_{2, n}(1)=\left(\frac{3}{4}\right)^{n+1}+\frac{n}{4}\left(\frac{3}{4}\right)^{n-1}-\frac{3}{2^{n+2}} \text { for } n>1 ; \\
& p_{2, n}(2)=1-\left(\frac{3}{4}\right)^{n+1}-\frac{1}{4}\left(\frac{3}{4}\right)^{n}-\frac{n}{4}\left(\frac{3}{4}\right)^{n-1} \text { for } n>1 ; \\
& p_{3, n}(0)=\frac{1}{16}\left(\frac{3}{4}\right)^{n}+\frac{9}{2^{n+4}}+\frac{6}{4^{n+2}} \text { for } n>2 ;
\end{aligned}
$$

The proof can be obtained in a standard way by the law of total probability application to following conditions

- shorter string consists of identical symbols,

- shorter string contains exactly two different symbols,

- shorter string contains three different symbols

and counting all the cases with the use of geometric series formula. Unfortunately for larger $n$ values calculations became too complicated and huge to get through.

The Monte Carlo simulation never gives exact information. Nevertheless, if lengths are small then computer can just find LCS for each couple of strings with given lengths and exactly calculate the probabilities by the formula $p_{n, m}(k)=\frac{X_{n, m}(k)}{4^{n+m}}$ where $X_{n, m}(k)$ is the total number of string couples with lengths $n$ and $m$ and LCS length $k$. Any modern $\mathrm{PC}$ allows to calculate probability mass function for any length with $m+n<16$ in a time less then one hour. But the performance time grows very fast, always more then 4 times with each added to total length symbol. Even if the Moore's Law continue its action, we shall never get a computational resource enough to proceed with $m+n=100$. So as the table will not grow much we publish it except lines of the values that 
TABle 1. Exact values $x_{m . n}(k)$ for $m=3, \ldots, 5$

\begin{tabular}{rrrrrrrr}
\hline$m$ & $n$ & $x_{m, n}(0)$ & $x_{m, n}(1)$ & $x_{m, n}(2)$ & $x_{m, n}(3)$ & $x_{m, n}(4)$ & $x_{m, n}(5)$ \\
\hline 3 & 3 & 105 & 570 & 333 & 16 & & \\
3 & 4 & 231 & 1797 & 1860 & 208 & & \\
3 & 5 & 537 & 5436 & 8715 & 1696 & & \\
3 & 6 & 1311 & 16131 & 36990 & 11104 & & \\
3 & 7 & 3345 & 47502 & 147441 & 63856 & & \\
3 & 8 & 8871 & 139713 & 562920 & 337072 & & \\
3 & 9 & 24297 & 411888 & 2083239 & 1674880 & & \\
3 & 10 & 68271 & 1219263 & 7530450 & 7959232 & & \\
3 & 11 & 195585 & 3626226 & 26726205 & 36560848 & & \\
3 & 12 & 568311 & 10834653 & 93468060 & 163564432 & & \\
3 & 13 & 1668057 & 32507028 & 322953267 & 716613472 & & \\
3 & 14 & 4930431 & 97874331 & 1104629190 & 3087533344 & & \\
4 & 4 & 453 & 4800 & 8742 & 2325 & & \\
4 & 5 & 951 & 12537 & 34737 & 16287 & 1024 & \\
4 & 6 & 2109 & 32688 & 125919 & 91572 & 9856 & \\
4 & 7 & 4911 & 85983 & 431556 & 452142 & 73984 & \\
4 & 8 & 11973 & 229512 & 1426380 & 2049063 & 477376 & \\
4 & 9 & 30471 & 623565 & 4602219 & 8740545 & 2780416 & \\
4 & 10 & 80589 & 1726080 & 14611437 & 35649990 & 15040768 & \\
4 & 11 & 220191 & 4864899 & 45895014 & 140496120 & 76959232 & \\
4 & 12 & 617493 & 13937016 & 143156802 & 538909425 & 377121088 & \\
4 & 13 & 1766391 & 40488273 & 444586053 & 2022505587 & 1785620992 & \\
& & & & & & & \\
5 & 5 & 1833 & 28890 & 118404 & 98340 & 14421 & \\
5 & 6 & 3759 & 67587 & 372240 & 478146 & 121980 & \\
5 & 7 & 8097 & 161496 & 1118523 & 2048547 & 803625 & 54016 \\
5 & 8 & 18231 & 395277 & 3276435 & 8083134 & 4546155 & \\
5 & 9 & 42873 & 992934 & 9470214 & 30124806 & 23194581 & 3283456 \\
5 & 10 & 105279 & 2562903 & 27226098 & 107738208 & 109830936 & \\
5 & 11 & 269457 & 6797844 & 78276255 & 373719987 & 491599113 & 123079168 \\
5 & 12 & 715911 & 18504897 & 225874791 & 1266831732 & 2105965533 & 677074432 \\
\hline & & & & & & & \\
\end{tabular}

consists of calculated by the theorem values. Table 1 and Table 2 contains the numbers $\frac{x_{n, m}(k)}{=} \frac{X_{n, m}(k)}{4}$ to avoid rounding errors completely. 
TABLE 2. Exact values $x_{m . n}(k)$ for $m=6, \ldots, 9$

\begin{tabular}{|c|c|c|c|c|c|c|c|c|c|c|c|}
\hline$m$ & $n$ & $x_{m, n}(0)$ & $x_{m, n}(1)$ & $x_{m, n}(2)$ & $x_{m, n}(3)$ & $x_{m, n}(4)$ & $x_{m, n}(5)$ & $x_{m, n}(6)$ & $x_{m, n}(7)$ & $x_{m, n}(8)$ & $x_{m, n}(9)$ \\
\hline 6 & 6 & 7221 & 144216 & 1028931 & 2022960 & 907179 & 82773 & 1024 & & & \\
\hline 6 & 7 & 14631 & 317769 & 2753415 & 7587861 & 5259105 & 821907 & 22528 & & & \\
\hline 6 & 8 & 30909 & 721704 & 7267770 & 26375340 & 26208204 & 6221289 & 283648 & & & \\
\hline 6 & 9 & 67839 & 1687911 & 19132338 & 87148998 & 117952467 & 39763023 & 2682880 & & & \\
\hline 6 & 10 & 154821 & 4067112 & 50587443 & 278159046 & 493461417 & 226129521 & 21182464 & & & \\
\hline 6 & 11 & 368151 & 10107261 & 134995695 & 867001353 & 1954801602 & 1180257714 & 147435520 & & & \\
\hline 7 & 7 & 28185 & 653256 & 6653442 & 25126110 & 26901270 & 7292628 & 449877 & 4096 & & \\
\hline 7 & 8 & 56751 & 1393707 & 16048740 & 77752320 & 118644660 & 49303485 & 5133393 & 102400 & & \\
\hline 7 & 9 & 118257 & 3069144 & 38981253 & 230646870 & 474140310 & 281409102 & 43935096 & 1441792 & & \\
\hline 7 & 10 & 254391 & 6959589 & 95813703 & 666472293 & 1767695262 & 1429288875 & 313426287 & 15056896 & & \\
\hline 7 & 11 & 566025 & 16255368 & 239097306 & 1896158694 & 6264656730 & 6664862241 & 1968372276 & 129900544 & & \\
\hline 8 & 8 & 109893 & 2818902 & 35794041 & 216361560 & 465327408 & 297936519 & 53028744 & 2348373 & 16384 & \\
\hline 8 & 9 & 220551 & 5910921 & 81120723 & 582733095 & 1662146217 & 1521603615 & 410485389 & 30288033 & 458752 & \\
\hline 8 & 10 & 454989 & 12762708 & 187198638 & 1542583476 & 5569004667 & 6924999531 & 2646395274 & 289326477 & 7143424 & \\
\hline 9 & 9 & 429513 & 11892570 & 173097576 & 1439627910 & 5342669283 & 6969837924 & 2872947741 & 357417270 & 11883861 & 65536 \\
\hline 9 & 10 & 860559 & 24709083 & 378466404 & 3527391882 & 16219427187 & 28529534742 & 16745716626 & 3120355458 & 170983179 & 2031616 \\
\hline
\end{tabular}


TABLE 3. Parameters of numeric simulation

\begin{tabular}{rrrc}
\hline$m+n$ & step of $n$ & step execution time & repetitions for step \\
\hline 32 & 1 & $20 \mathrm{sec}$ & $1111275-1173205$ \\
512 & 8 & $20 \mathrm{sec}$ & $49334-161188$ \\
8192 & 128 & $20 \mathrm{~min}$ & $15240-185462$ \\
131072 & 2048 & $200 \mathrm{~min}$ & $535-4391$ \\
2097152 & 262144 & $10000 \mathrm{sec}$ & $2-5$ \\
\hline
\end{tabular}

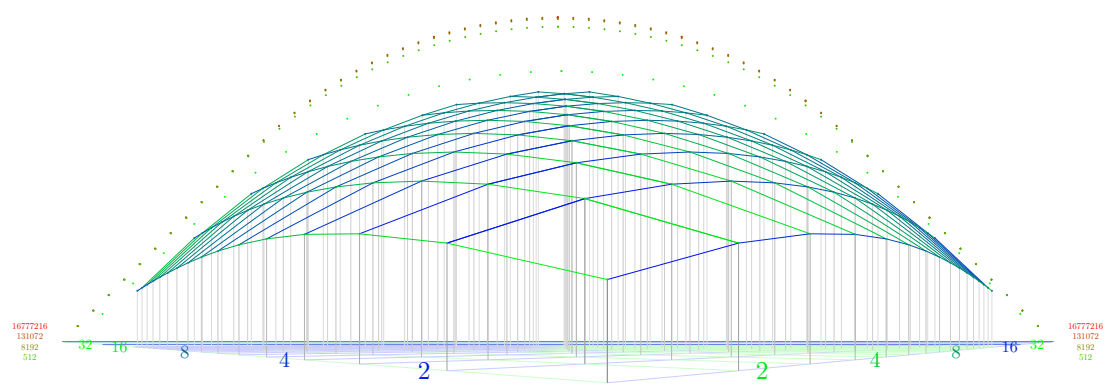

Figure 1. Expected value of LCS as a function of the sequence lengths

\section{Design and interpretation of expected value and variance graphs}

We believe that the function $E\left(L_{m, n}\right)$ of two string length is concave and grows nearly linear with lengths grows. This is a reason to use projective geometry to draw graphs in linear perspective and see the limit values in infinity as the horizon.

The calculated exact values does not represent behaviour on large lengths. So numeric simulation was used to get a full picture. It used fixed total length $n+m$ and a fixed step on $n$ with fixed stepwise execution time. Table 3 shows the given parameters of numeric simulation and number of repetitions for each step. The graph of expected value on the Picture 1 use the projective transform $\left(\frac{2 x-8 y}{2+5 x+5 y}, \frac{10 z-1}{2+5 x+5 y}\right)$ mapping numbers to centimeters. The point of view was selected close to a vertex $(-0.25,-0.25,0.1)$ of approximate asymptotic cone by a series of iterations aimed to get images of far points proportionally with respect to logarithms of numbers closer to some limit line. Our experiment detects $\gamma_{4}=0.6542$ to be compatible with the published in [5] more precise value $\gamma_{4}=0.654361$ obtained in very smart numeric simulation. 


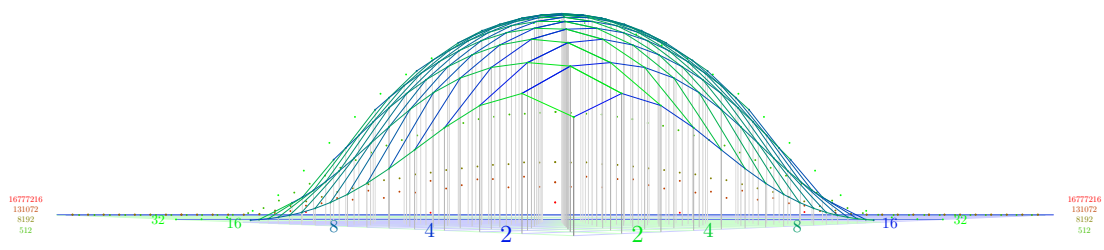

FIgURE 2. Standard deviation of LCS as a function of the sequence lengths

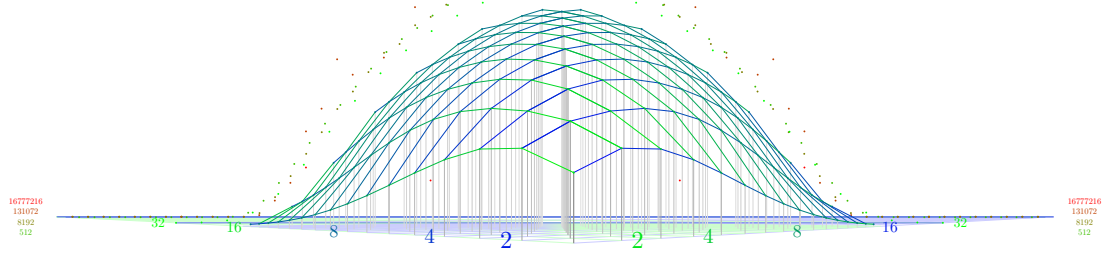

Figure 3. Standard deviation of LCS scaled by $\sqrt[4]{m+n}$

The graph show unexpected structure of dotted consisting from central circle arc extended by two line segments. So the graph visualy looks smoothly combined from two plains $z=x, z=y$ and surface $z=$ $s-\sqrt{2} r+\sqrt{r^{2}-s^{2}}$ where $\left.s=\frac{x+y}{2}, r=(\sqrt{2}+1)(s-e(s))\right)$ and the diagonal function $e(n)=E\left(L_{n, n}\right)$ is monotonic, concave and unknown, but for large $m$ and $n$ it is close to linear and therefore surface is close to asymptotic right circular cone.

The graph of variance on the Picture 2 use another projective transform $\left(\frac{x-1.1 y}{7+x+y}, \frac{12 z-0.4}{7+x+y}\right)$ mapping numbers to centimeters to reflect differences from conical form. We see that variance grows more slow then linear. The asymptotic (non-circle) similar to cone surface appears visible on the Picture 3 for magnified variance $\sqrt[4]{m+n} \cdot V\left(L_{m, n}\right)$ shown under $\left(\frac{x-1.1 y}{7+x+y}, \frac{6 z-0.6}{7+x+y}\right)$ transform.

\section{References}

[1] R. Durbin, S. Eddy, A. Krogh, G. Mitchison. Biological sequence analysis: probabilistic models of proteins and nucleic acids, Cambridge University Press, 1998, 370 c. $\uparrow^{201}$ 
[2] J. G. Reich, H. Drabsch, A. Däumler. «On the statistical assessment of similarities in DNA sequences», Nucleic acids research, 12:13 (1984), c. $5529-5543 . \uparrow^{201}$

[3] K. Ning, K.P. Choi. Systematic assessment of the expected length, variance and distribution of Longest Common Subsequences, 2013, arXiv: 1306.4253. ${ }^{201}$

[4] V. Chvátal, D. Sankoff. «Longest Common subsequences of two random sequences», J. Appl. Probability, 12:2 (1975), c. 306-315. $\uparrow^{201}$

[5] R. Bundschuh. «High precision simulations of the longest common subsequence problem», The European Physical Journal B-Condensed Matter and Complex Systems, 22:4 (2001), c. 533-541. $\uparrow^{202,205}$

Submitted by

dr E. P. Kurshev

About the author:

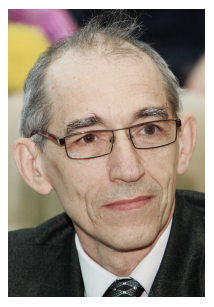

\section{Sergej Vital'evich Znamenskij}

Chair of Mathematics in the Ailamazyan Pereslavl University, head of laboratory in Ailamazyan Program Systems Institute of RAS. Research interests migrate from research in Functional Analysis, Complex Analysis and finite-dimensional Projective Geometry (analogues of Convexity) to the foundations of Collaborative Software Development.

e-mail:

svz@latex.pereslavl.ru

Sample citation of this publication:

S. V. Znamenskij. "A picture of common subsequence length for two random strings over an alphabet of 4 symbols", Program systems: theory and applications, 2016, 7:1(28), pp. 201-208.

URL:

http://psta.psiras.ru/read/psta2016_1_201-208.pdf 
УДК 004.416

С. В. Знаменский. Картина наиболъшей длины общих подпоследователъностей пары случайных строк 4 буквенного алфавита.

АннотАция. Наибольшая длина (LCS) общей подпоследовательности пары случайных конечных последовательностей из 4 букв рассмотрена как случайная функция от длин $m$ и $n$ этих двух последовательностей. Представлены таблицы точных значений вероятностей для всех пар конкретных длин в диапазоне $2<m+n<19$.

Графики зависимости математического ожидания и дисперсии показаны в линейной перспективе, позволяющей просматривать на горизонте поведение при растущих длинах. Для иллюстрации поведения при больших значениях длин на этих же графиках показаны результаты численного эксперимента для больших значений $m+n=32,512,8192$ и 131072 . Представленный график зависимости математического ожидания от $m$ и $n$ выглядит имеющим асимптотический прямой круговой конус. Дисперсия выглядит растущей как $(n+m)^{\frac{3}{4}}$.

Ключевые слова и фразы: сходство строк, выравнивание последовательностей, случайные общие подпоследовательности, LCS, метрика Левенштейна.

\section{Пример ссылки на эту публикащию:}

С. В. Знаменский. «Картина наибольшей длины общих подпоследовательностей пары случайных строк 4 буквенного алфавита», Программные системы: теория и приложения, 2016, 7:1(28), с. 201-208. (Англ).

URL:

$$
\text { http://psta.psiras.ru/read/psta2016_1_201-208.pdf }
$$

